\title{
Feeding who, when and how, dysphagia in advanced dementia
}

\begin{abstract}
Dysphagia is a growing health issue especially in aging population. It can lead to a high variety of complications. Here we present the case of an old woman who suffered from dementia and dysphagia. She was admitted to our ward and was diagnosed with aspiration pneumonia. We obtained a full swallowing evaluation, after that we decided not to position a percutaneous gastric tube. Oral nutrition is at least as good as enteral for the outcomes of death, aspiration pneumonia, functional status in patients suffering from dysphagia related to dementia.
\end{abstract}

Keywords: advanced dementia, dysphagia, enteral feeding, oral feeding
Volume 4 Issue 3 - 2020

\author{
Luca Matteucci,' 'Valeria Giosia,' Martino \\ Brenna,' Lisa Serati,' Agnese Massafra,' \\ Aurora Smeriglia,' Antonio Luca Brucato ${ }^{2}$ \\ 'Resident Physician at Complex Operative Unit of Internal \\ Medicine, Dipartimento di Scienze Biomediche e Cliniche, \\ Università degli Studi $d$ Milano, Italy \\ ${ }^{2}$ Chief of Medicine at Complex Operative Unit of Internal \\ Medicine, Dipartimento di Scienze Biomediche e Cliniche, \\ Università degli Studi d Milano, Italy
}

\begin{abstract}
Correspondence: Antonio Luca Brucato, Chief of Medicine at Complex Operative Unit of Internal Medicine, Dipartimento di Scienze Biomediche e Cliniche, Università degli Studi d Milano, Ospedale Fatebenefratelli e Oftalmico, Piazzale Principessa Clotilde 3, Milano (2012I), Italy, Tel 00390263632500 , Email antonio.brcato@unimi.it
\end{abstract}

\begin{abstract}
Correspondence: Luca Matteucci, Resident Physician at Complex Operative Unit of Internal Medicine, Dipartimento di Scienze Biomediche e Cliniche, Università degli Studi d Milano, Ospedale Fatebenefratelli e Oftalmico, Piazzale Principessa Clotilde 3, Milano (20I2I), Italy, Tel 00390263632500 , luca, Emailmattecci@unimi.it
\end{abstract}

Received: June 06, 2020 | Published: June 17, 2020

\section{Case description}

An 85 years old woman was taken to the emergency room for dyspnea. She suffered from dysphagia due to vascular dementia with severe cognitive impairment and not otherwise specified parkinsonism. She was on enteral feeding via nasogastric tube because of an episode of aspiration pneumonia that had occurred two months before. She also suffered from chronic atrial fibrillation, hypothyroidism, dysphagia, and was under several medications, among others L-DOPA + benserazide, oxazepam and pregabalin. She lived at home and enteral feeding was managed by her relatives, who reported an episode of aspiration that occurred some hours before admission, after an attempt to increase enteral solution infusion rate to $80 \mathrm{ml} /$ hour.

At the admission the patient was dyspnoic and arterial blood gas analysis was consistent with severe respiratory failure (pO2 33 mmHg; pCO2 49 mmHg; sO2 71\%). Therefore, a bronchial aspiration was performed, and oxygen therapy was started. A full blood count showed no leukocytosis, but $\mathrm{C}$ reactive protein was elevated up to 271 $\mathrm{mg} / \mathrm{L}$. A chest X-ray showed a right lung pulmonary consolidation; consequently, aspiration pneumonia diagnosis was made, and the patient was admitted to our ward. She was treated with intravenous ceftriaxone plus metronidazole for seven days.

The patient's caregiver had been previously instructed to administer the enteral feeding solution at a rate of at least $80 \mathrm{ml} /$ hour, in order to avoid severe malnutrition and possibly death. When evaluated by our nurses, the patient was able to swallow semisolid food, although discontinuously. Therefore, we obtained a full endoscopic swallowing evaluation. Penetration aspiration scale (PAS) scores were $1 / 8$ for semi-solids, $2 / 8$ for solids, and 3/8 for liquids; Dysphagia Outcome and Severity Scale (DOSS) was $4 / 7$. We obtained an evaluation by nutritionists who prescribed a mixed feeding regimen with semi-solid food by mouth accompanied by protein concentrates and water via nasogastric tube. We avoided positioning of percutaneous gastric tube (PEG). The patient was discharged and returned home after 12 days on a mixed diet, oral and enteral, with focus on a careful oral handfeeding.

\section{Discussion}

Dysphagia is a growing health issue especially in aging population. It can be associated with a huge variety of medical conditions typical of elderly such as stroke, dementia, Parkinson's disease, tumors, and iatrogenic interventions. Prevalence of dysphagia in people suffering from dementia can be extremely high, up to $45 \%$ in institutionalized patients, and can lead to increased hospital admission rates and overall mortality. The appearance of dysphagia in advanced dementia is an ominous sign, linked to a bad prognosis. Aspiration pneumonia is also a serious threat and is associated with a $30 \%$ hospital mortality and to a $50 \%$ six-month mortality. Dysphagia and mental impairment can lead to malnutrition and low albumin level, which is associated to a poorer prognosis..$^{1,2,3}$ Therefore, management of dysphagia in elderly suffering from cognitive impairment is a quite delicate issue. ${ }^{1}$ Enteral tube feeding is a common intervention during hospitalizations, used 
to manage dysphagia and poor food intake, especially in advanced dementia. However, at present there is no evidence to support its efficacy in this condition. Older adults suffering from advanced dementia who underwent an enteral feeding tube placement showed higher mortality compared to those who did not. ${ }^{2}$ In addition, tube feeding can be associated to recurrent aspiration due to difficulty in managing oral secretion. Other complications include higher infection rates, increased risk of pressure ulcers development, tube malfunctions or use of physical and chemical restraint to improve feeding tube tolerability. ${ }^{2,4}$

Among older adults with advanced dementia a careful oral hand-feeding is preferable to enteral nutrition. ${ }^{5}$ Recently, scientific society guidelines stressed the importance of providing oral nutrition using attractive food in a pleasant environment, with good nursing care to manage and prevent malnutrition in patients with dementia. Artificial nutrition is therefore suggested only in patients with transient insufficiency of oral intake in order to overcome a critical situation. The patient prognosis as well as the natural history of the disease must be taken into account. ${ }^{6}$ A popular solution for our patient would be PEG positioning to prevent further aspiration. However, we suggested partial oral feeding re-introduction given her medical history and her actual swallowing ability. Careful hand feeding should be offered to people with advanced dementia. Oral nutrition is at least as good as enteral for the outcomes of death, aspiration pneumonia, functional status, and comfort in these patients. Besides, tube feeding is associated with agitation, greater use of physical and chemical restraints, tube-related complications and increased risk of pressure ulcers. Hospitals should promote a fully informed decision-making and should not exert pressure on individuals or health providers to introduce tube feeding.

\section{Acknowledgments}

None.

\section{Conflicts of interest}

The author declares there is no conflict of interest.

\section{Funding}

None.

\section{References}

1. Bosch X, Formiga F, Cuerpo S, et al. Aspiration pneumonia in old patients with dementia. Prognostic factors of mortality. Eur J Intern Med. 2012;23(8):720-726.

2. Sampson EL, Candy B, Jones L. Enteral tube feeding for older people with advanced dementia. Cochrane database Syst Rev. 2009;(2):CD007209.

3. Brucato AL, Ferrari A, Tiraboschi M, et al. Three-month Mortality in Permanently Bedridden Medical Non-Oncologic Patients. The BECLAP Study (Permanently BEdridden, Creatinine CLearance, Albumin, Previous Hospital Admissions Study). Eur J Intern Med. 2020;72:60-66.

4. Teno JM, Gozalo P, Mitchell SL, et al. Feeding tubes and the prevention or healing of pressure ulcers. Arch Intern Med. 2012;172(9):697-701.

5. American Geriatrics Society Ethics Committee and Clinical Practice and Models of Care Committee. American Geriatrics Society feeding tubes in advanced dementia position 91 statement. $J$ Am Geriatr Soc. 2014;62(8):1590-1593.

6. Volkert D, Chourdakis M, Faxen-Irving G, et al. ESPEN guidelines on nutrition in dementia. Clin Nutr. 2015;34(6):1052-1073. 